\title{
Preset delay broadcast: a protocol for fast information dissemination in vehicular ad hoc networks (VANETs)
}

\author{
Salvador Gonzalez and Victor Ramos ${ }^{*}$
}

\begin{abstract}
Vehicular ad hoc networks (VANETs) have been proposed in order to assist the driver on the road. There are multiple applications where VANETs are needed, for example, proposing routes to reach a given destination, cooperating for traffic management, or preventing the driver of dangers on the road. In this paper, we focus on message broadcast for driver safety. Such broadcasting must be fast and reliable such that all the vehicles in a certain area receive the message as fast as possible. There are several proposals in the literature of broadcast protocols for critical messages in VANETs. In order to get a wide view of the different techniques to broadcast a message, we evaluate a set of protocols representing one or more broadcast techniques. Moreover, we propose PDB, a preset delay broadcast protocol with a fixed delay for vehicles attempting to retransmit a warning message, which provides a fast and reliable dissemination. We show that by adequately setting the waiting time for the relay candidates, we can significantly reduce the delay to cover a given area, while at the same time preserving a good reliability. Moreover, we model different techniques to broadcast an emergency packet in a VANET such as count-based, geographical, distance-based, and opportunistic, and thus we implement a subset of state-of-the-art protocols that implement one or more of those techniques. Finally, our research shows that stopping beacon transmissions when a warning message is detected does not provide a significant performance improvement. Nonetheless, by allowing a continuous channel access, we prove that the performance of any protocol might be greatly increased.
\end{abstract}

Keywords: Vehicular ad hoc networks, Intelligent transportation systems, Wireless access in vehicular environments (WAVE), Critical messages, Broadcast storm

\section{Introduction}

VANETs are intended to offer a wide range of services such as warnings for traffic signal violation, intersection collision, highway merge assistance, approaching vehicle emergency, and curve speed just to mention a few. Organizations have put great effort to standardize vehicular networks; for example, the Association of Radio Industries and Businesses (ARIB STD-T109) [1] for Japan, the European Telecommunications Standards Institute (ETSI, TC ITS) [2], and the International Organization for Standardization (ISO/TC 204/WG 18) [3] for Europe and some other parts of the world. In this work, we focus on wireless access in vehicular environments (WAVE), which

*Correspondence: vicman@xanum.uam.mx

Universidad Autónoma Metropolitana, Department of Electrical Engineering,

San Rafael Atlixco 186, 09340, Iztapalapa, Mexico City, Mexico offers an architecture to provide safety and non-safety services. Figure 1 shows a general view of such architecture. The communication is carried out by the IEEE 802.11p standard [4], which is intended to provide efficient mechanisms for a part of the medium access control MAC and physical PHY layers.

Multichannel operation is carried out according to the IEEE 1609.4 standard [5]. As shown in Fig. 2, there are seven channels, where six of them are service channels $(\mathrm{SCH})$ and one is a control channel $(\mathrm{CCH})$. The purpose of $\mathrm{SCH}$ is to provide services for critical and non-critical applications, as well as for IP traffic. The $\mathrm{CCH}$ allows to exchange management frames, short WAVE messages, and messages for critical applications. Each channel has a bandwidth of $10 \mathrm{MHz}$; however, SCH 174 and SCH 176 may be used as a single $20-\mathrm{MHz}$ bandwidth channel. The 


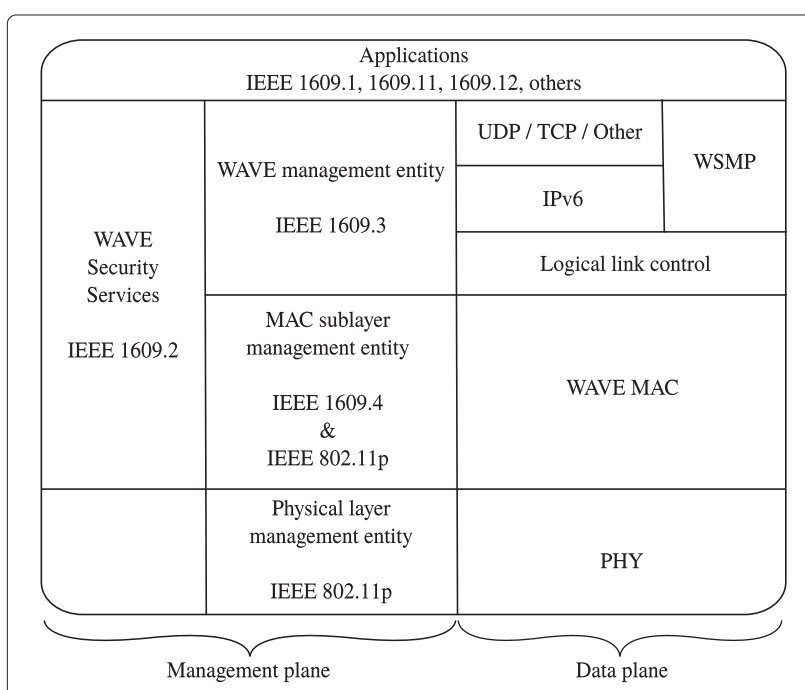

Fig. 1 WAVE system architecture

same applies to SCH 180 and SCH 182. The WAVE system allows four different ways for channel access, which are shown in Fig. 3. The continuous access type guarantees channel access at any time for the $\mathrm{CCH}$ or $\mathrm{SCH}$. The alternating access type allocates $50 \mathrm{~ms}$ either to the $\mathrm{CCH}$ or to one of the SCH. In this work, we assess both types of channel access. We refer the reader to [6] for a further explanation on WAVE systems.

We focus mainly on the broadcast of messages for driver safety. Such a broadcast process must be fast and reliable such as all the vehicles in a certain area receive the message as fast as possible; however, two main problems may arise. On one hand, VANETs suffer from the broadcast storm problem occurring when all the vehicles or a large subset of them rebroadcast a packet. When this occurs, the medium access contention increases, which produces a high delay on message delivery, message collisions, and hidden terminals. On the other hand, VANETs

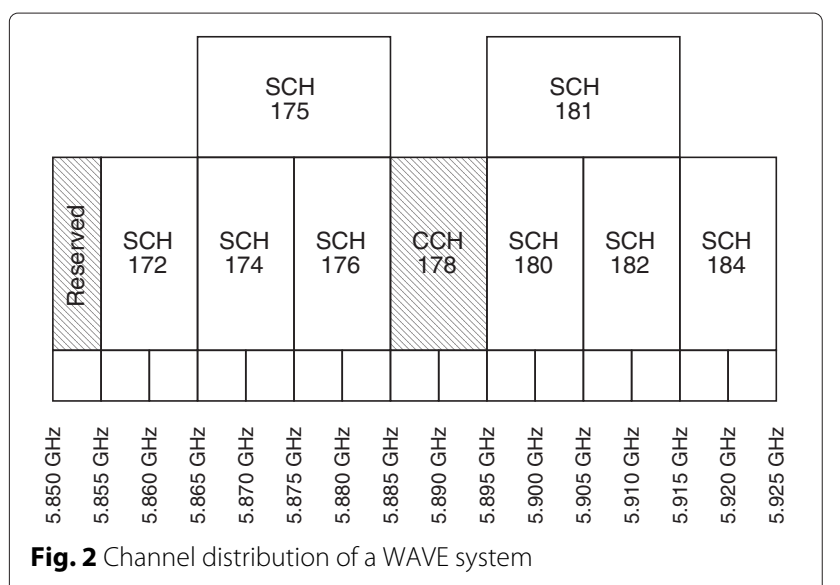

also exhibit the network disconnection problem, which is due to the vehicles' mobility that causes network partitioning and becomes eventually the cause for the loss of communication.

The rest of this paper is structured as follows. Section 2 reviews the related work about the proposed solutions for the broadcast storm and network disconnection problems. Section 3 describes the protocols we analyze in this work as well as the considerations we take to compare them. Then, we propose in Section 4 our preset delay broadcast (PDB) protocol that exhibits a fast message dissemination and a high reliability. Section 5 depicts the scenarios evaluated along with the implementation of state-of-the-art protocols in order to compare their performance with PDB. Next, we present in Section 6 the results we obtain after an extensive campaign of simulations. Finally, Section 7 sketches our conclusions and future work.

\section{Related work}

The research on VANETs currently covers a wide set of subjects, where among the most important ones we may find the work on routing protocols, multimedia services, multicast, and broadcast transmission. We provide below a brief overview of the state of the art on each of these directions in order to point out where our work is focused.

Regarding routing protocols, Spyropoulos et al. [7] identify a taxonomy of opportunistic protocols for delaytolerant networks (DTNs). The aim of such work is to provide guidelines for designers so that one may choose a network routing protocol well suited for applications. The main identified relevant parameters for the routing process in that work are network density, node heterogeneity, and mobility patterns. In [8], the authors focus on energy saving and propose DRSS (directional routing and scheduling scheme), which is a routing protocol for DTNs that uses a Nash Q-learning approach to optimize energy efficiency along with network congestion, buffer, and delay occupation. The proposed scheme is implemented on the NS-2 simulator to show its ability to improve energy efficiency and data delivery ratio with such a learning mechanism to predict the network environment. Moreover, the work in [9] focuses also on energy-efficient protocols and proposes a data collection method that the authors call Energy-Efficient, DelayAware, and Lifetime-Balancing Data Collection (EDAL) protocol. The authors prove that the problem formulation is NP-hard. Even if EDAL is proposed for wireless sensor networks, the mechanism might be useful for different types of networks like VANETs.

Concerning multimedia services for VANETs, in [10], Zhou et al. propose a distributed media service to solve the trade-off among content dissemination, cache update, and fairness for P2P-based (peer-to-peer) vehicular 


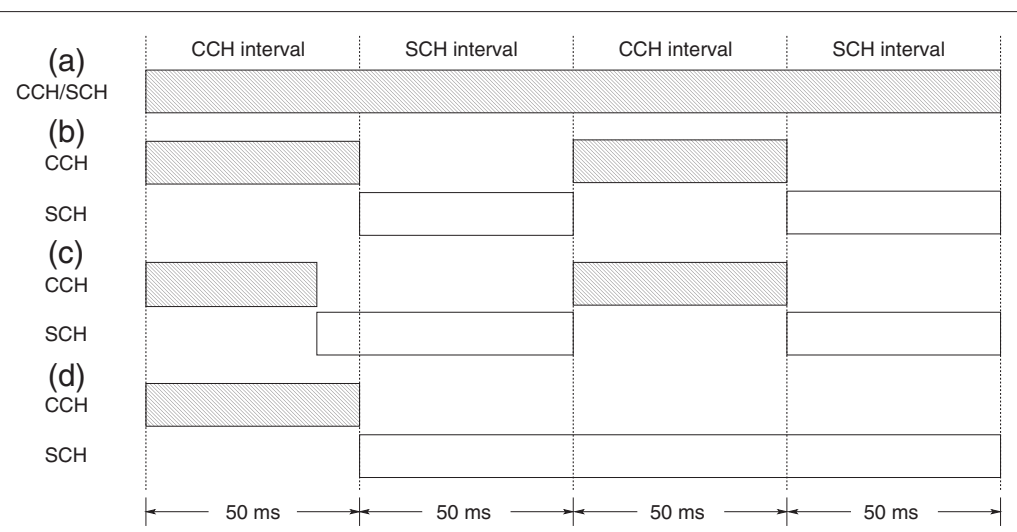

Fig. 3 Types of channel access in a WAVE system. a Continuous. b Alternating. c Immediate. d Extended

networks. The proposal focuses on user satisfaction rather than on quality of service and considers media-aware distribution along with opportunistic transmission. Besides, the work in [11] provides a great overview of multimedia services for cloud-based VANETs. The main technological challenges of providing cloud-based services are discussed by considering communication infrastructure, cloud taxonomies, network integration, and a wide set of applications.

Considerable work has been done on multicast protocols for mobile ad hoc networks (MANETs). Protocols for MANETs may often be adapted to operate on VANETs, so contributions on this direction may be very useful to provide efficient multicast mechanisms for both types of networks. For example, in [12], the authors propose a multi-constrained QoS multicast protocol that uses a genetic algorithm. The proposed protocol therein takes into account parameters such as crossover, mutation, and population size. Also, in [13, 14], the authors propose CodePipe, which is a reliable multicast protocol designed to exhibit very good performance on energy efficiency, throughput, and fairness.

Finally, broadcast protocols for VANETs aim to offer a set of applications for driver safety. Such applications must deliver critical messages as fast as possible. However, each application has its own requirements; furthermore, applications may start broadcasting under different circumstances, like for example, those defined in [15] such as Time-Limited Periodic Messages Broadcasting on Event (TLPMBonE), Periodic Triggered by Vehicle Mode (PTbyVM), V2X Co-operative awareness (V2xCoA), Time-Limited Periodic Messages Geocasting on Event (TLPMGonE), Authoritative Message Triggered by Traffic Management Entity (AMTbyTME), Temporary Messages Broadcasting on Event (TMBonE), Temporary Messages Geocasting on Event (TMGonE), and Periodic Co-operative Awareness Broadcast (PCoABcast). We summarize in Table 1 cooperative road safety applications along with the just cited communication modes, their minimal broadcast frequency, and their corresponding critical time.

In this paper, our focus is on broadcast protocols for critical messages. Such protocols intend to solve at least one of the two main problems faced by VANETs, i.e., broadcast storm and network disconnection. Next, we summarize the main broadcast techniques for critical messages in the literature $[16,17]$. Moreover, the main contribution of this work is our PDB protocol that provides a fast and reliable message dissemination. PDB is described later in Section 4.

\subsection{Flooding}

When a vehicle receives a message for the first time, it must rebroadcast it. Therefore, each vehicle receiving a message for the first time must repeat this procedure until all the vehicles in a desired area receive the message. This type of broadcast exhibits high reliability in a sparse network. However, for dense networks, flooding may suffer from the broadcast storm problem. Furthermore, the collision probability increases as well as the hidden terminal problem. These algorithms are inspired on those used for mobile ad hoc networks, like the one in [18].

\subsection{Probabilistic}

In this type of protocols, each vehicle that receives successfully the message decides whether to rebroadcast it or not according to a probability distribution. These protocols reduce the medium contention as well as the number of collisions and redundant messages. However, when these protocols are used in sparse networks, it is possible that some vehicles do not receive the message. The speed adaptive probabilistic flooding algorithm [19] and the weighted $p$-persistent, slotted 1-persistent, and the slotted $p$-persistent are examples of probabilistic protocols [20]. In [21], an adaptive probabilistic protocol is proposed where, on one hand, vehicles in a dense network 
Table 1 Cooperative road safety applications

\begin{tabular}{|c|c|c|c|}
\hline Application & Communication mode & Mininum frequency & Critical time \\
\hline Emergency electronic brake lights & TLPMBonE & $10 \mathrm{~Hz}$ & $<100 \mathrm{~ms}$ \\
\hline Safety function out of normal condition warning & TLPMBonE & $1 \mathrm{~Hz}$ & $<100 \mathrm{~ms}$ \\
\hline Emergency vehicle warning & PTbyVM & $10 \mathrm{~Hz}$ & $<100 \mathrm{~ms}$ \\
\hline Slow vehicle warning & PTbyVM & $2 \mathrm{~Hz}$ & $<100 \mathrm{~ms}$ \\
\hline Motorcycle warning & $\mathrm{V} 2 \times \mathrm{CoA}$ & $2 \mathrm{~Hz}$ & $<100 \mathrm{~ms}$ \\
\hline Vulnerable road user warning & $\mathrm{V} 2 \times \mathrm{COA}$ & $1 \mathrm{~Hz}$ & $<100 \mathrm{~ms}$ \\
\hline Wrong way driving warning & TLPMBonE & $10 \mathrm{~Hz}$ & $<100 \mathrm{~ms}$ \\
\hline Stationary vehicle warning & TLPMBonE & $10 \mathrm{~Hz}$ & $<100 \mathrm{~ms}$ \\
\hline Traffic condition warning & TLPMBonE, TLPMGonE, or AMTbyTME & $1 \mathrm{~Hz}$ & N/A \\
\hline Signal violation warning & TMBonE or AMTbyTME & $2 \mathrm{~Hz}$ & $<100 \mathrm{~ms}$ \\
\hline Roadwork warning & TMBonE or TMGonE & $10 \mathrm{~Hz}$ & $<100 \mathrm{~ms}$ \\
\hline Decentralized floating car data & TLPMBonE & 1 to $10 \mathrm{~Hz}$ & N/A \\
\hline Overtaking vehicle warning & $\mathrm{V} 2 \times \mathrm{COA}$ & $10 \mathrm{~Hz}$ & $<100 \mathrm{~ms}$ \\
\hline Lane change assistance & $\mathrm{V} 2 \times \mathrm{COA}$ & $10 \mathrm{~Hz}$ & $<100 \mathrm{~ms}$ \\
\hline Pre-crash sensing warning & $\mathrm{V} 2 \times \mathrm{COA}$ & $10 \mathrm{~Hz}$ & $<50 \mathrm{~ms}$ \\
\hline Cooperative glare reduction & $\mathrm{V} 2 \times \mathrm{COA}$ & $2 \mathrm{~Hz}$ & $<100 \mathrm{~ms}$ \\
\hline Across traffic turn collision risk warning & $\mathrm{V} 2 \times \mathrm{CoA}$ & $10 \mathrm{~Hz}$ & $<100 \mathrm{~ms}$ \\
\hline Merging traffic turn collision risk warning & $\mathrm{V} 2 \times \mathrm{COA}$ & $10 \mathrm{~Hz}$ & $<100 \mathrm{~ms}$ \\
\hline Cooperative merging assistance & $\mathrm{V} 2 \times \mathrm{COA}$ & $10 \mathrm{~Hz}$ & $<100 \mathrm{~ms}$ \\
\hline Hazardous location notification & TMBonE or AMTbyTME & $10 \mathrm{~Hz}$ & N/A \\
\hline Intersection collision warning & PCoABcast & $10 \mathrm{~Hz}$ & $100 \mathrm{~ms}$ \\
\hline Cooperative forward collision warning & V2xCoA Unicast & $10 \mathrm{~Hz}$ & $100 \mathrm{~ms}$ \\
\hline Collision risk warning from RSU & TLPMBonE & $10 \mathrm{~Hz}$ & $100 \mathrm{~ms}$ \\
\hline
\end{tabular}

have a low probability of rebroadcasting messages and, on the other hand, in a sparse network they have a higher rebroadcast probability.

\subsection{Counter-based}

Vehicles in a VANET that implement this type of protocols decide to rebroadcast a message based on the number of times they have received the same message. The main idea is as follows: when a vehicle receives a message for the first time, it waits a time $t$ before rebroadcasting it. When a vehicle receives the same message and exceeds a given threshold, then such a vehicle cancels the rebroadcasting process. Thus, if the timer expires, the vehicle does not rebroadcast the message since it has been rebroadcast for other vehicles. If the timer expires and the threshold has not been reached, then the vehicle must rebroadcast the message in order to increase the coverage of the message. This type of technique has been combined with the probabilistic scheme in [22], where the authors propose to rebroadcast a message according to a probabilistic distribution, while at the same time the protocol manages a counter to cancel the procedure if the message has been received too many times. Moreover, an adaptive counter-based protocol is proposed in [23]. Here, nodes rebroadcast a message considering the number of times that the message is received along with the inter-arrival times. In [24], the authors propose a dynamic counterbased protocol. In this protocol, the authors use a different threshold depending on how many vehicles are close to the node. A node trying to rebroadcast a message that is within a dense area of vehicles will have a smaller threshold than when it is in a sparse area.

\subsection{Distance-based}

In this type of protocols, the decision of whether to rebroadcast a message or not depends on the distance between the transmitter and the receiver. Hence, only the vehicles located at a distance greater than a given threshold rebroadcast the message. These protocols are highly dependent on the threshold, which often causes a variable performance. In [25], the authors propose a protocol where the vehicles attempting to retransmit a message wait $t$ seconds, where $t$ depends on the distance between the transmitter and the receiver as well as on the communication range. In [26], a protocol that selects the relay by partitioning the communication range is proposed; here, 
the vehicle located in the farthest partition retransmits the message.

\subsection{Neighbor knowledge}

In order to decide whether to rebroadcast a packet or not, this type of protocols take advantage of the location and movement of the neighbors. Thus, a vehicle may decide if there is a better relay or if it is the best candidate. However, for sparse networks, this algorithm might lack the necessary information to make the best choice. In [27, 28], the authors make use of connected dominating sets (CDS) to propose a protocol aimed to reduce unnecessary retransmissions. With CDS, a graph is created in order to select the minimum number of nodes to cover $100 \%$ of their corresponding neighbor nodes.

\subsection{Opportunistic}

The main idea of opportunistic protocols is to take advantage of the opportunities inherent to the broadcast process. Thus, the farthest vehicle that receives successfully the message has more chances to rebroadcast the packet, hence the broadcast process may complete with few hops. In [29], the authors assign a high priority to the farthest vehicles so they have a shorter waiting time to rebroadcast the message. In [30], we extend and improve OB-VAN [31] where the farthest vehicles have more chances to rebroadcast the message. We adopt the OB-VAN's main idea for the selection process. Once a vehicle receives a packet, intervals of the same length are generated to receive or transmit short acknowledgments. When a vehicle is within the reception interval and receives an acknowledgment, then such vehicle stops its corresponding selection process meaning that there is a better relay. The main difference between OB-VAN and our previous proposal, Fast-OB-VAN, is that the latter transmits the emergency packet rather than a short acknowledgment. Fast-OBVAN achieves a faster message dissemination by carefully selecting what it sends.

\section{Study cases}

\subsection{Protocols analyzed}

In order to account with a general view of the different techniques to broadcast a message, we select a set of state-of-the-art protocols implementing one or more techniques listed above. Thus, we implement a simple flooding protocol since such a dissemination technique represents the worst case. In a similar way, we also implement a simple counter-based protocol with a threshold equal to three messages. Moreover, we implement the bounding algorithm originally proposed for MANETs [32], which uses the counter and distance techniques; the main motivation to implement this protocol is that it may potentially cover a given area with few hops. Regarding the neighbor knowledge approach, we implement the
non-GPS data dissemination protocol [28], which uses the number of neighbors of each vehicle to decide which vehicle rebroadcasts a message and, ideally, covers all the vehicles. An additional feature of this protocol is that it does not require to know the vehicles' location to select the best relay; thus, a Global Positioning System (GPS) is not needed. A GEographical Data Dissemination for Alert Information protocol gives an overview of the performance of geographical algorithms. Such a technique makes a partition of the communication range, which causes that only the vehicles in some partitions are able to retransmit the messages. We address opportunistic protocols with OppCast, which takes advantage of the distance between the transmitter and receiver. We also implement our previous contribution Fast-OB-VAN. As with $O p p$ Cast, Fast-OB-VAN also uses the distance to select the best relay.

Finally, we evaluate the performance of our proposal that is discussed in Section 4.

\subsection{Algorithm efficiency}

For the first step of the campaign of simulations, we evaluate all the protocols just described under standard conditions. Even if some mechanisms are originally proposed to work with a different MAC layer or under conditions other than those stated by the IEEE 1609.X or the IEEE $802.11 \mathrm{p}$ standards, we implement all the protocols according to the IEEE standards. Thereby, we are able to analyze the performance of the different mechanisms considered under the same conditions. With such an assessment, we may identify the advantages and disadvantages of the different techniques evaluated. Thus, we have a message exchange as shown in Fig. 4.

\subsection{Stopping beacons}

From the previous study case, we can see that warning messages and beacons must contend for the medium. Such contention may induce a high delay for warning messages, collisions, or hidden terminals. Thus, when a vehicle receives or needs to transmit a warning message, we stop the beacons in order to reduce the contention for this type of messages as shown in Fig. 5. Such an improvement allows to reduce the medium contention as the vehicles detect the warning message.

\subsection{Continuous access}

Finally, we evaluate the previous study case with continuous access for the $\mathrm{CCH}$ in order to reduce the delay added during channel switching so as to get a faster dissemination. Whenever a control channel switches to one of the service channels, safety messages experience a delay equal to the operation time of the service channel. Such a delay may occur more than one once during the whole 


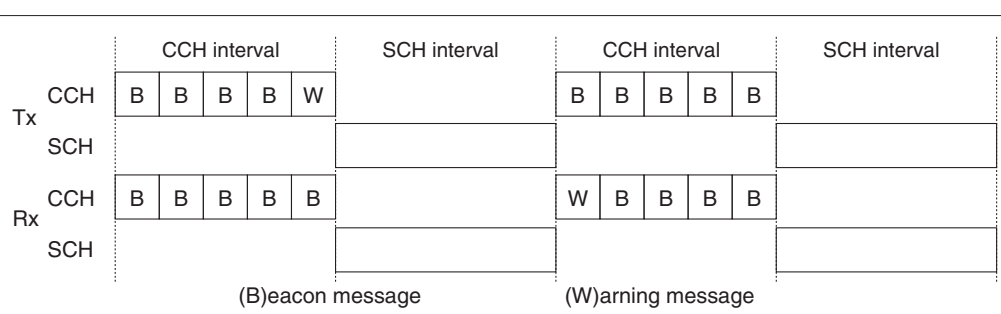

Fig. 4 An example of the possible messages for scenario 1

dissemination process. Continuous access removes the additional delay. This study case is shown in Fig. 6.

\section{Preset delay broadcast (PDB) protocol}

Our goal in this work is to design a protocol that exhibits low delay and high reliability. Thus, we propose our PDB protocol with a fixed delay for vehicles attempting to retransmit a warning message in order to provide a fast message dissemination. The PDB protocol is fully compliant with the IEEE 802.11p standard. Thus, we guarantee an efficient message dissemination that closely follows the standard specifications. Moreover, we assume that all the vehicles are equipped with a GPS, and thus they are aware of their location. Such assumption is considered by most of the broadcast protocols for VANETs because WAVE systems require the exchange of this type of information.

In a WAVE system, all the vehicles share their location and movement by means of beacons. Accordingly, a vehicle needing to transmit a warning packet knows how far its neighbors are located. With such information, the source may sort the neighbors according to the distance. Therefore, the source may decide which neighbors are the best candidates to retransmit the message among the ten farthest ones. Thus, the farthest neighbors must have a lower delay to retransmit the packet. In this work, we set a delay of $0.5 \mathrm{~ms}$ for the farthest neighbor, $1 \mathrm{~ms}$ for the second farthest neighbor, $1.5 \mathrm{~ms}$ for the third farthest neighbor, and so on until the tenth farthest neighbor that has a delay of $5 \mathrm{~ms}$. Hence, when a vehicle transmits a warning packet, the farthest ten neighbors are added to the warning packet. Additionally, the distance of the farthest neighbor is added too.
We provide in Fig. 7 a flowchart that depicts the reception of messages in our PDB protocol. The detailed explanation is as follows: when a vehicle receives a warning message, it checks the list of neighbors added to such message. If the receiver finds its own ID in the list, it waits the corresponding delay as we described above. If the receiver does not find its own ID in the list, then it checks the distance to the farthest node. On one hand, if a vehicle does not find its own ID and the distance to the source is lower than the one added at the end of the list, then such a vehicle will have a random delay between 7 and $10 \mathrm{~ms}$. This ensures that the message dissemination will continue if there is no vehicle in the list retransmitting the packet. On the other hand, if a vehicle does not find its own ID and the distance to the source is greater than the one added to the packet, then such a vehicle will attempt to immediately retransmit the packet. This allows the farthest vehicles that have not been added to the neighbors list to make a faster retransmission.

An example of the data added to the warning packet is shown in Fig. 8. In this example, the neighbor with ID 8 has a delay of $0.5 \mathrm{~ms}$, the neighbor with ID 2 has a delay of $1 \mathrm{~ms}$, the neighbor with ID 3 has a delay of $1.5 \mathrm{~ms}$, and so on. A random delay between 7 and $10 \mathrm{~ms}$ is set for a vehicle receiving the broadcast packet, which is not included in the list and with a distance lower than $290 \mathrm{~m}$. In a similar way, a vehicle receiving the broadcast packet not being included in the list with a distance greater than $290 \mathrm{~m}$ will have a delay of $0 \mathrm{~ms}$.

When a vehicle retransmits a warning message, it will replace the list of IDs with its corresponding list according to its neighbors. In addition, in order to deal with network

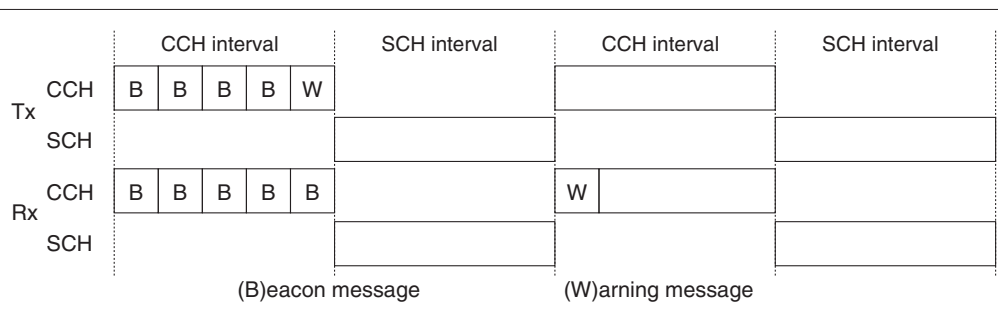

Fig. 5 An example of the possible messages for scenario 2 


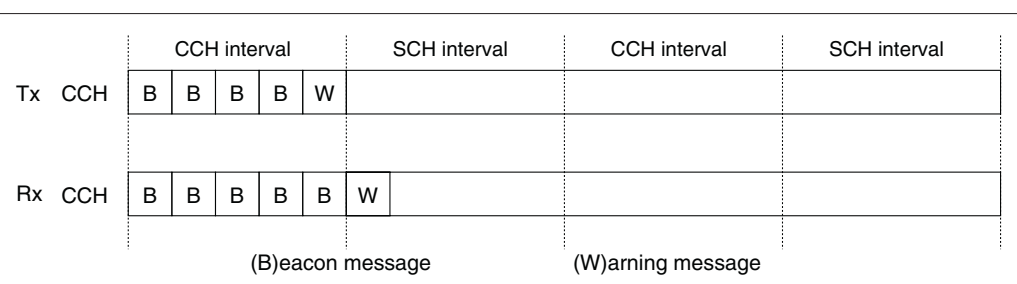

Fig. 6 An example of the possible messages for scenario 3

disconnections, it is possible to implement the mechanism store-carry-forward as described in [33]. Finally, when a vehicle receives the warning message for the second time, then it cancels the retransmission and ignores future receptions of that message. Algorithm 1 provides the pseudo-code of our proposed PDB protocol.

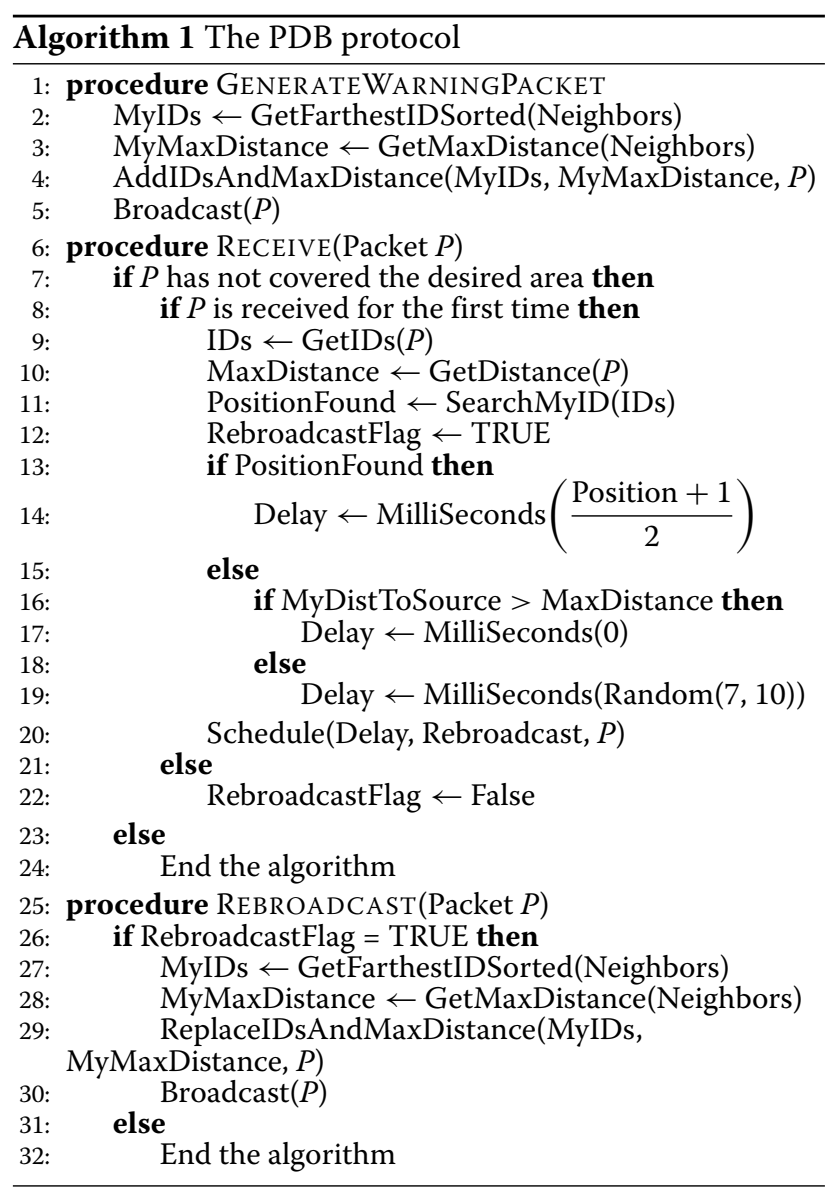

\section{Simulation scenario and performance parameters}

We describe in this section how we assess the performance of the different protocols we select. Thus, we use SUMO [34] to generate a three-lane highway scenario with vehicles moving in the same direction as shown in
Fig. 9. When a source vehicle broadcasts a message, it is disseminated in the opposite direction of the flow of vehicles up to a distance of $1 \mathrm{~km}$. The length of the highway is a relevant consideration in this scenario. Hence, we consider a 3-km-length highway in order to focus on the vehicles located at the center. Thus, we do not have to deal with the vehicles at the start of the road where they are static; similarly, we do not deal with vehicles at the end of the road where they are stopped, which eases the message exchange in the simulator.

Table 2 lists the parameters describing the configuration of the vehicle movement. We consider a total of eight mobility traces, with different vehicle densities. Thus, for the network simulation, the set $\{17,34,51,71,83,102$, $120\}$ represents the average number of vehicles in the target area. Notice that the vehicle's maximum speed is set to $4.5 \mathrm{~m} / \mathrm{s}$, which might be considered low for vehicular mobility. However, even if the vehicles travel at $80 \mathrm{~m} / \mathrm{s}$, such vehicles only move $4 \mathrm{~m}$ in the 50 -ms duration of the $\mathrm{CCH}$. Furthermore, vehicles travel at similar speeds and in the same direction. Therefore, there is not a significant change in the network topology during the transmission. Nevertheless, the network topology changes during the entire simulation, thus having different experiments in this way. Consequently, in order to generate different vehicle densities, we consider a low vehicle speed so as to produce vehicular congestion.

We implement the algorithms in the NS-3 simulator [35], which implements the PHY and MAC layers according to the IEEE 802.11p standard. In order to fit better to the WAVE systems, we use a module provided by Junling $\mathrm{Bu}$ [36]. Such module provides multichannel operation. With such an improvement, we define one $\mathrm{CCH}$ and one $\mathrm{SCH}$. On the $\mathrm{CCH}$, vehicles transmit beacons along with emergency packets. Even with a $\mathrm{SCH}$, vehicles do not transmit anything on that channel, which is necessary to stop the $\mathrm{CCH}$ operation every $50 \mathrm{~ms}$ as recommended for WAVE systems. In Table 3, we show the general configuration for the network. Finally, we consider that one experiment is carried out each time that a vehicle broadcasts a packet, and then the corresponding protocol rebroadcasts it to cover the target area. Thus, we execute 4000 experiments per mobility trace, which makes a total of 32,000 experiments per protocol. 


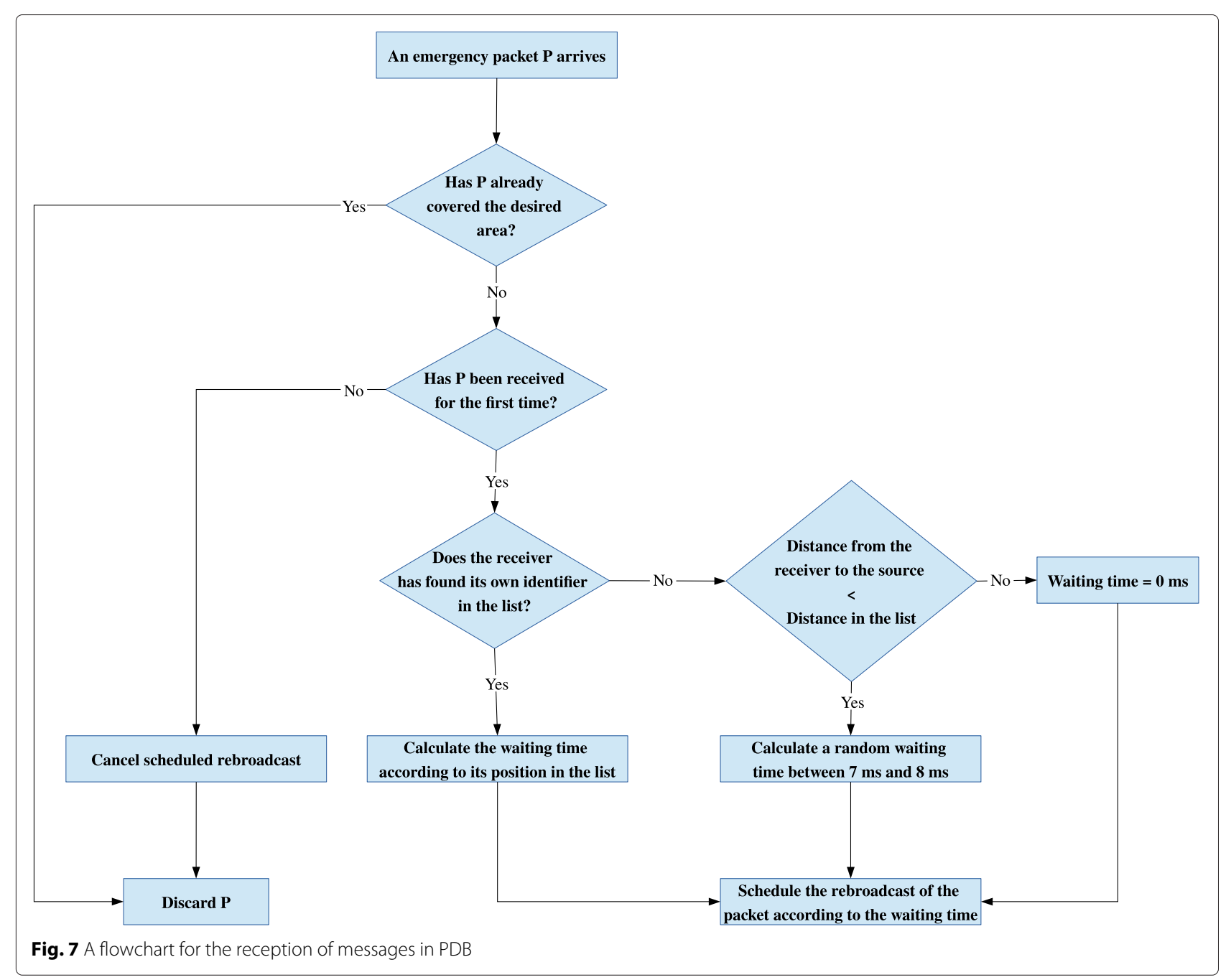

\section{Results}

In this section, we present the results we obtain after an extensive campaign of simulations. We focus on four parameters: the delay to cover a target area, the number of packet retransmissions, the number of vehicles having correctly received the broadcast packet within a certain area, and the number of times that the protocol completes correctly. For the first three parameters, the measurements are taken only when the protocols complete correctly.

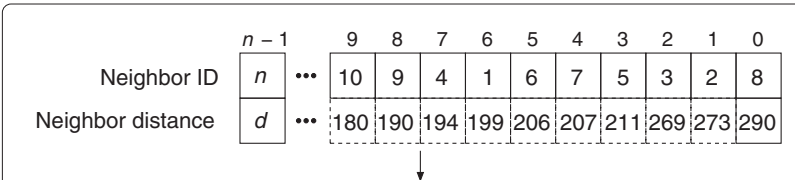

$$
\begin{aligned}
& \begin{array}{|c|c|c|c|c|c|c|c|c|c|c|}
10 & 9 & 8 & 7 & 6 & 5 & 4 & 3 & 2 & 1 & 0 \\
\hline 290 & 10 & 9 & 4 & 1 & 6 & 7 & 5 & 3 & 2 & 8 \\
\hline
\end{array}
\end{aligned}
$$

Fig. 8 An example of the data added to the warning packet

\subsection{Average delay}

Figure 10 plots the average delay as a function of the number of nodes. For each experiment, we measure the delay as follows. The source adds a time stamp when transmitting a message, thus all the vehicles located at a distance greater than or equal to $1 \mathrm{~km}$ report the delay by computing the time difference between the current time in the receiver and the time stamp in the message. Even if more than one vehicle reports a delay value, we only take into account the first report.

Protocol efficiency. For the first study case, flooding is the fastest protocol covering $1 \mathrm{~km}$. This is because such a protocol does not have to deal with a waiting time to rebroadcast the message. Then, $\mathrm{PDB}$ and bounding closely exhibit delays similar to flooding. On one hand, since PDB presets a delay value, the waiting time to rebroadcast a packet is controlled, which allows to obtain a small delay. On the other hand, bounding allows at least three of the farthest vehicles to rebroadcast the packet. With a slightly higher delay, we find the count-based and NonGPSDD 


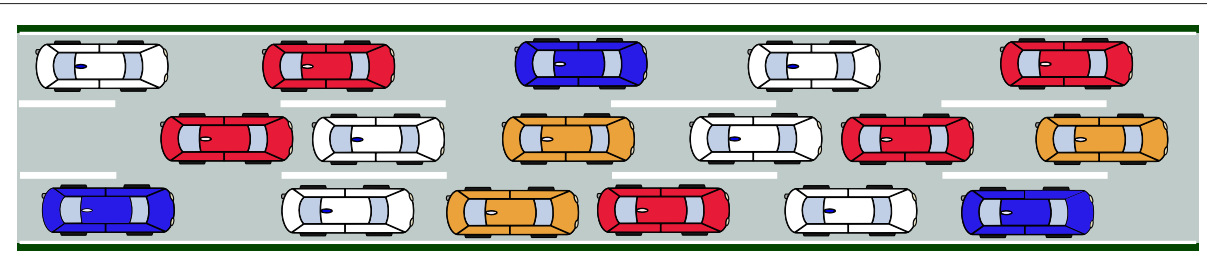

Fig. 9 Linear VANET scenario

protocols exhibiting a similar behavior. However, notice how the delay of NonGPSDD decreases when vehicle density increases. Then, the protocols following in delay performance are Fast-OB-VAN, closely followed by GEDDAI and OppCast.

Stopping beacons. For the second study case, we stop beacon transmissions. There is a decrease on delay; however, it is quite small. Thus, it might be hard to see a difference between plots in Fig. 10 (top-left) with those in Fig. 10 (center-left). Hence, it is clear that stopping beacons does not contribute in a significant way to reduce the delay needed to cover a given area.

Continuous access. In our third study case, we allow vehicles to continuously access the channel. Thus, we may see in Fig. 10 (bottom-left) that all the algorithms exhibit a delay lower than the previous study cases. Such a decrease on the average delay is because the message does not have to wait for channel switching. Thus, the message is sent as soon as needed.

Hence, only taking into account delay, the best choice to broadcast a message is flooding, followed by our PDB protocol and bounding. The analysis we provide in the following subsections will show if such a behavior is preserved.

\subsection{Packet retransmissions}

In order to measure this parameter, we focus on the average number of vehicles rebroadcasting a packet. We consider that a given vehicle rebroadcasts a packet after a successful reception and the broadcast algorithm retransmits such packet. Furthermore, the packet retransmission

Table 2 Vehicle's configuration

\begin{tabular}{ll}
\hline Parameter & Value \\
\hline Acceleration & $1.1 \mathrm{~m} / \mathrm{s}^{2}$ \\
Deceleration & $5.0 \mathrm{~m} / \mathrm{s}^{2}$ \\
minGap & $0.1 \mathrm{~m}$ \\
Initial lane & Random \\
Initial speed & Random \\
maxSpeed & $4.5 \mathrm{~m} / \mathrm{s}$ \\
Car-following model & SUMOKrauß \\
\hline
\end{tabular}

rate is calculated by dividing the total number of vehicles retransmitting the packet by the total of number of vehicles successfully receiving it.

Protocol efficiency. We can see in Fig. 10 (top-right) that bounding is the protocol requiring the lower number of retransmissions. Then, Fast-OB-VAN, GEDDAI, NonGPSDD, and OppCast show a similar performance. These last algorithms along with PDB exhibit a similar performance for a low number of nodes. However, notice how the performance of PDB increases starting at 34 nodes; this is because we assign a lower waiting time to the relay candidates. Since the difference to rebroadcast the packet between vehicles is $0.5 \mathrm{~ms}$, they cannot opportunely receive the packet and rebroadcast it. Nevertheless, when the vehicle density increases, the retransmission rate decreases, which is a desirable feature for all the algorithms. Then, we can see that starting with 17 nodes, the counter-based protocol incurs in a high rebroadcasting rate, then such a measure quickly decreases; however, notice that its performance slowly increases when vehicle density increases as well. Finally, with flooding, all the vehicles that successfully receive the packet must rebroadcast. Thus, we get a $100 \%$ of the vehicles rebroadcasting the packet.

Stopping beacons. Similarly as it happens with the average delay, with our second study case that considers to stop beacons, the difference in performance is quite small; thus, Fig. 10 (top-right) and Fig. 10 (center-right) are similar. Hence, we can conclude again that stopping beacons does not contribute in a significant way to reduce the percentage of vehicles rebroadcasting a packet.

Table 3 Network configuration

\begin{tabular}{ll}
\hline Parameter & Value \\
\hline Tx power & $20 \mathrm{~dB} \mathrm{~m}$ \\
Communication range & $300 \mathrm{~m}$ \\
Propagation loss model & Nakagami \\
Propagation delay & Constant speed propagation \\
& delay model \\
$\mathrm{SCH}$ and CCH duration & $50 \mathrm{~ms}$ \\
Beacon generation rate & $10 \mathrm{~Hz}$ \\
Emergency packet generation rate & $1 / 10 \mathrm{~s}^{-1}$ \\
\hline
\end{tabular}



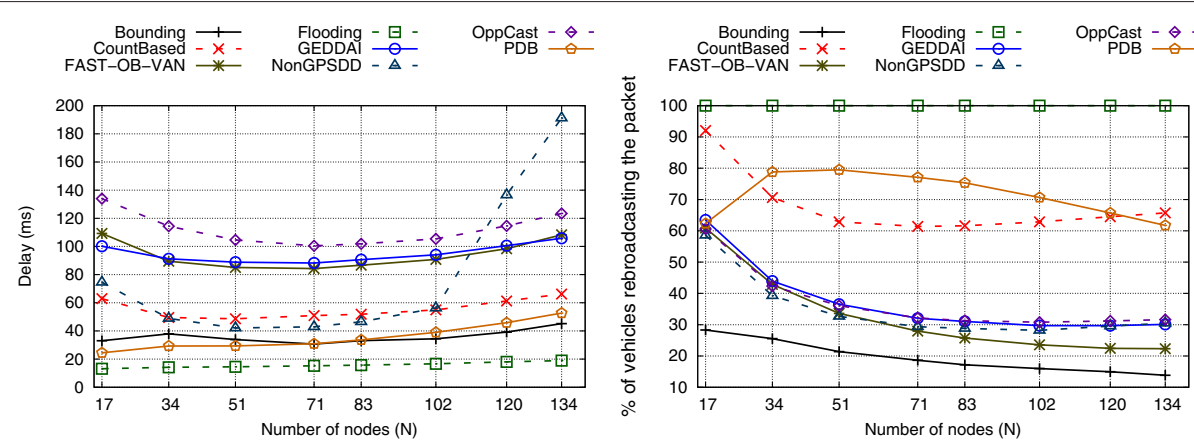

$\begin{array}{rr}\text { Bounding-PB - } & \text { GEDDAI-PB - }- \\ \text { CountBased-PB - } & \text { NonGPSDD-PB - } \\ \text { FAST-OB-VAN-PB - } \\ \text { Flooding-PB - }-\end{array}$
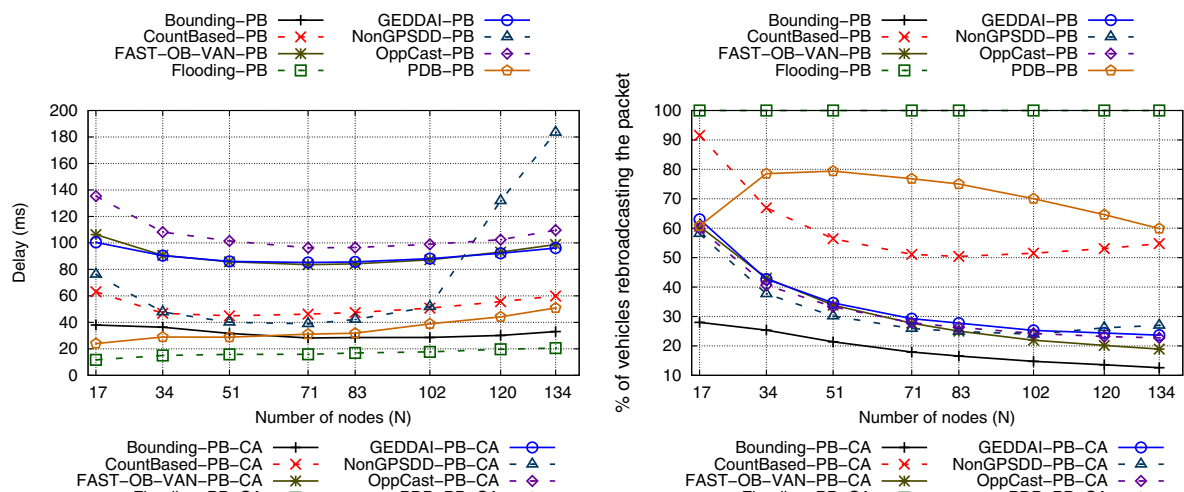

FB-VAN-PB-CA $\rightarrow$
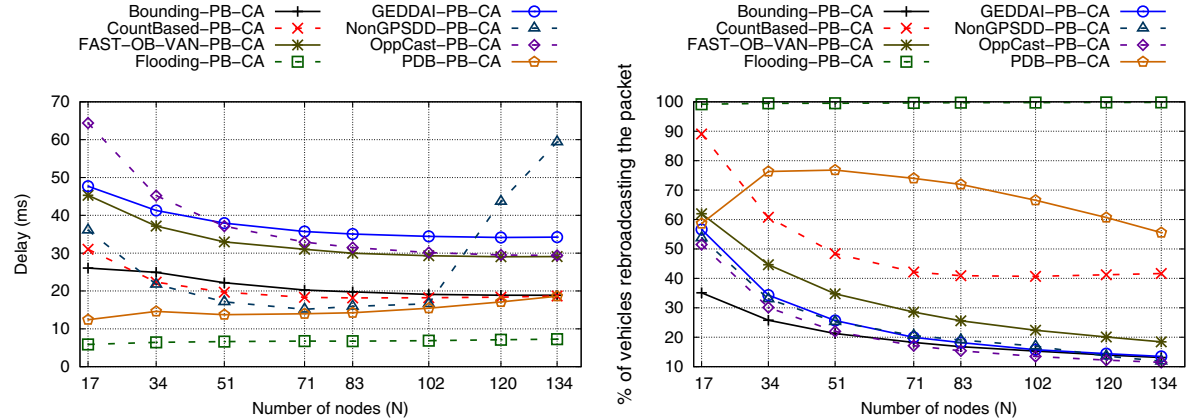

Fig. 10 Left: average delay as a function of the number of nodes in study cases one (top), two (center), and three (bottom). Right number of vehicles rebroadcasting the packet in study cases one (left), two (center), and three (right)

Continuous access. Our third study case considers continuous access to the channel. Thus, we may appreciate in Fig. 10 (bottom-right) a slight decrease on the percentage of vehicles rebroadcasting the packet. We can explain such a decrease with the fact that the broadcast process is not interrupted for channel switching.

\subsection{Number of times the protocol completes correctly}

We consider that the protocol completes correctly if the packet successfully arrives at a vehicle located at least $1 \mathrm{~km}$ away from the vehicle that created such packet. If no vehicle satisfies such condition, then we consider that the protocol does not complete correctly. With this information, we can determine how reliable the protocols are.

Protocol efficiency. Figure 11 (top-left) plots the number of times that the protocol completes correctly. Notice how bounding exhibits the worst performance. Thus, even if for the two previous parameters the bounding protocol performs well, for this parameter, bounding only completes correctly $25 \%$ of times in the best case. Such a low performance is because bounding only allows the vehicles within a certain area to contend to be a relay; the rest of the vehicles never attempt to rebroadcast the message. Thus, if there are no vehicles in the threshold, or due to collisions there is no vehicle able to successfully retransmit the packet, then the protocol will not be able to reach the $1-\mathrm{km}$ distance. In addition, if we modify such behavior to allow the vehicles out of the threshold area to attempt to retransmit the packet with a greater delay to give priority to the vehicles in the threshold area, then bounding would show a behavior similar to GEDDAI but with a different choice in the priority areas. The rest of the protocols show a similar performance when the density of vehicles increases. While with a low vehicle density, each protocol faces problems to achieve a better performance. It is 


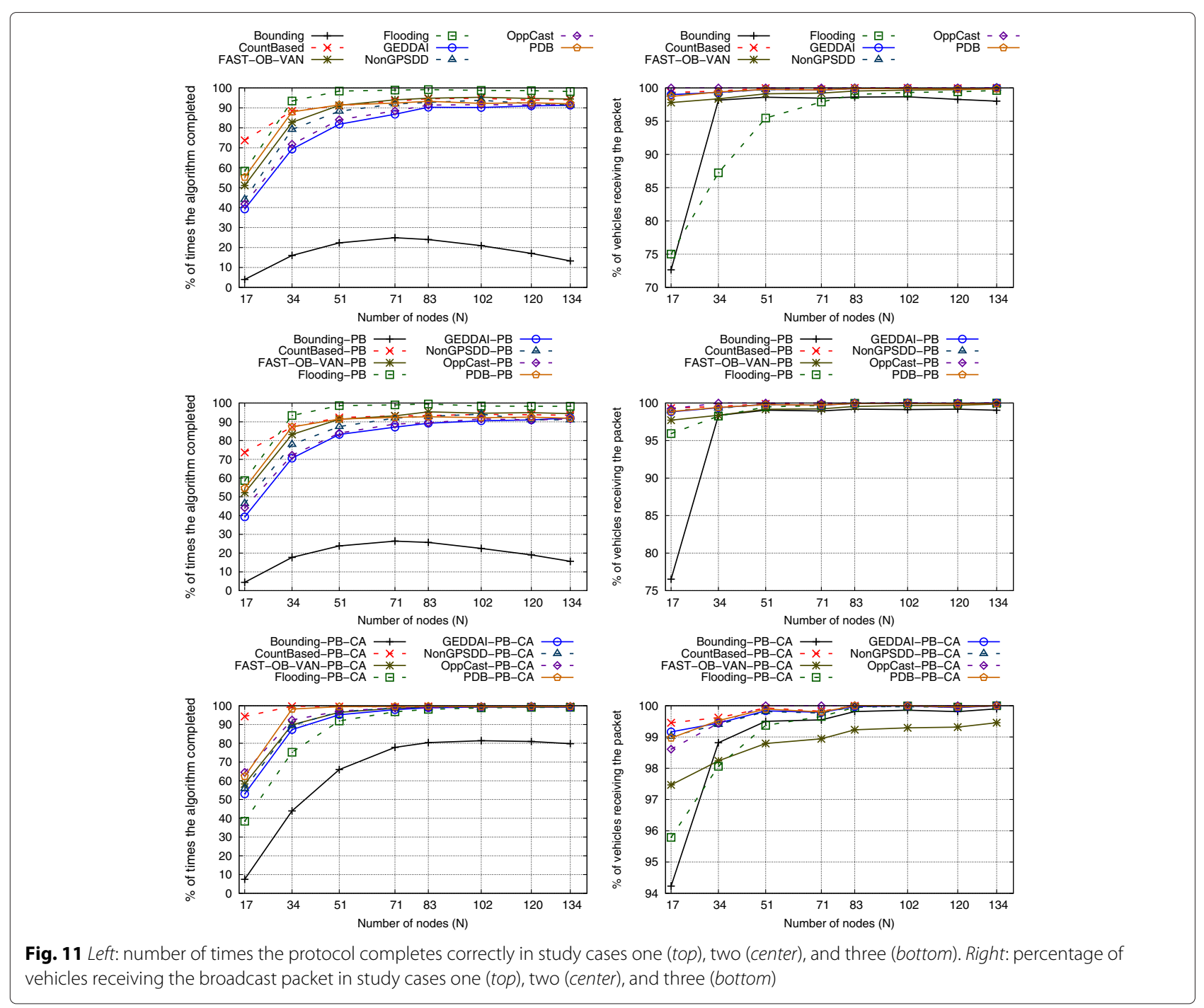

important to notice that in this work, we do not address network partitioning. However, each protocol might easily implement a store-carry-and-forward procedure like the one detailed in [29].

Stopping beacons. As what happens with previous cases, by comparing Fig. 11 (top-left) and Fig. 11 (center-left), we clearly see that stopping beacons does not significantly contribute to improve the percentage of times that the protocol completes correctly.

Continuous access. Figure 11 (bottom-left) shows a slight performance increase for most of the protocols. However, bounding has a drastic increase on reliability. This implies that channel switching highly impacts the performance of the bounding protocol. Nevertheless, bounding still has the worst reliability among the rest of the protocols.

\subsection{Percentage of vehicles receiving the broadcast packet} The last parameter we consider in our evaluation is the average percentage of vehicles receiving the broadcast packet. This is calculated by dividing the number of vehicles that successfully receive the packet by the total number of vehicles in the area of interest.

Protocol efficiency. The first study case is plotted in Fig. 11 (top-right). Even if flooding exhibits a good performance in terms of delay as well as on the percentage of time that the protocol completes correctly, in this case, flooding shows a low percentage of vehicles receiving the broadcast packet when the vehicle density is low. In the same way, bounding shows a similar behavior when the vehicle density is low. The rest of the protocols show an excellent percentage of vehicles receiving the broadcast packet, which is above $97 \%$.

Stopping beacons. Unlike the previous parameters, notice in Fig. 11 (center-right) how the flooding protocol exhibits a performance increase. The rest of the protocols also show a small performance increase.

Continuous access. We can see in Fig. 11 (bottom-right) a performance improvement for the bounding protocol. 
For this study case, all the protocols reach more than $94 \%$ of vehicles correctly receiving the broadcast packet.

\section{Conclusions}

In this work, we made an extensive analysis of several protocols proposed in the literature for message dissemination in VANETs under three study cases. In order to exhibit low delay and high reliability to broadcast warning messages, we proposed a protocol that sets the waiting time for relay candidates. We shown that by doing this, we can significantly reduce the delay needed to cover a given area. Furthermore, even if our PDB protocol incurs in several retransmissions, they are significantly reduced as the vehicle density increases. Additionally, PDB offers a high reception rate and high reliability when covering an interest area.

In order to model different techniques as countbased, geographical, distance-based, and opportunistic, we implemented a subset of protocols using one or more of such techniques. We may see that flooding provides the lowest delay to cover a given area. However, its message dissemination suffers from the broadcast storm problem since all the vehicles receiving the broadcast packet must retransmit it, and this reduces the reception rate because of collisions or hidden terminals. Bounding combines the count-based and distance-based techniques. In a vehicular environment, bounding exhibits a very low propagation delay with a few number of retransmissions. However, since such a protocol only allows to contend for being a relay among the vehicles within a certain area, it has success only very few times; i.e., it is unreliable. NonGPSDD is self-dependent from the use of a GPS; this is an advantage for VANETs. Even if this protocol shows good performance in general, the delay tends to quickly increase when the density of vehicles increases as well. The geographical protocol (GEDDAI) along with the opportunistic Fast-OB-VAN and OppCast perform well in most of the metrics considered. However, these protocols exhibit the highest delay to cover the area of interest. Finally, the counter-based and our proposed PDB protocol show similar performance. On one hand, one important difference is the percentage of vehicles rebroadcasting the packet; the counter-based protocol incurs on fewer retransmissions than PDB for most of the vehicle densities. Even so, the counter-based protocol tends to increase the number of retransmissions along with the density of vehicles, whereas PDB tends to decrease the number retransmissions with a higher density of vehicles. On the other hand, PDB exhibits lower delay than counter-based.

We have also shown that stopping the beacons when a warning message is detected does not represent a major performance improvement. However, allowing a continuous channel access is beneficial for all the protocols to a large extent.
As a future work, we will analyze the performance of our PDB protocol in a more complex scenario. Our main interest is to study the behavior of PDB on highways and urban areas, as well as with multiple sources generating emergency messages. The main motivation for doing so is to identify the performance difference when PDB is executed on a highway environment since the vehicle speed is normally faster than when vehicles move in an urban scenario. Also, for urban scenarios, vehicle density is greater than for highway scenarios. Furthermore, we would like to assess the performance of PDB under multiple warning message sources. With such an assessment, we will account with the necessary information to know if the protocol still exhibits good performance with a greater network load.

\section{Competing interests}

The authors declare that they have no competing interests.

\section{Acknowledgements}

The authors are grateful to the National Council of Science and Technology (CONACyT: Consejo Nacional de Ciencia y Tecnología) for supporting this work.

Received: 22 January 2016 Accepted: 16 April 2016

Published online: 26 April 2016

\section{References}

1. $700 \mathrm{MHz}$ Band Intelligent Transport Systems, ARIB STD-T109 (2012). http://www.arib.or.jp/english/html/overview/std-t109.html. Accessed 22 April 2016

2. Intelligent Transport Systems (ITS); Communications Architecture (2010). http://www.etsi.org/technologies-clusters/technologies/intelligenttransport. Accessed 22 April 2016

3. ITS Co-operative systems ISO/TC 204/WG 18 (2010). http://www.iso.org/ iso/iso_technical_committee\%3Fcommid\%3D54706. Accessed 22 April 2016

4. IEEE Standard for information technology_local and metropolitan area networks - specific requirements. Part 11: Wireless LAN Medium Access Control (MAC) and Physical Layer (PHY) Specifications Amendment 6: Wireless Access in Vehicular Environments. Institute of Electrical \& Electronics Engineers (IEEE) (2010). doi:10.1109/ieeestd.2010.5514475

5. IEEE Standard for Wireless Access in Vehicular Environments (WAVE)-multi-channel operation. Institute of Electrical \& Electronics Engineers (IEEE) (2011). doi:10.1109/ieeestd.2011.5712769

6. IEEE Guide for Wireless Access in Vehicular Environments (WAVE) - architecture. Institute of Electrical \& Electronics Engineers (IEEE) (2011). doi:10.1109/ieeestd.2014.6755433

7. T Spyropoulos, RNB Rais, T Turletti, K Obraczka, A Vasilakos, Routing for disruption tolerant networks: taxonomy and design. Wireless Netw. 16(8), 2349-2370 (2010). doi:10.1007/s11276-010-0276-9

8. Y Zeng, KXiang, D Li, AV Vasilakos, Directional routing and scheduling for green vehicular delay tolerant networks. Wireless Netw. 19(2), 161-173 (2012). doi:10.1007/s11276-012-0457-9

9. Y Yao, Q Cao, AV Vasilakos, EDAL: An energy-efficient, delay-aware, and lifetime-balancing data collection protocol for heterogeneous wireless sensor networks. IEEE/ACM Trans. Netw. 23(3), 810-823 (2015). doi:10.1109/tnet.2014.2306592

10. L Zhou, Y Zhang, K Song, W Jing, AV Vasilakos, Distributed media services in P2P-based vehicular networks. IEEE Trans. Vehic. Technol. 60(2) 692-703 (2011). doi:10.1109/tvt.2010.2102782

11. M-K Jiau, S-C Huang, J-N Hwang, AV Vasilakos, Multimedia services in cloud-based vehicular networks. IEEE Intell. Transportation Syst. Mag. 7(3), 62-79 (2015). doi:10.1109/mits.2015.2417974

12. Y-S Yen, H-C Chao, R-S Chang, A Vasilakos, Flooding-limited and multi-constrained QoS multicast routing based on the genetic algorithm 
for MANETs. Math. Comput. Modell. 53(11-12), 2238-2250 (2011) doi:10.1016/j.mcm.2010.10.008

13. P Li, S Guo, S Yu, AV Vasilakos, in Proceedings of the IEEE Infocom. CodePipe: an opportunistic feeding and routing protocol for reliable multicast with pipelined network coding. (Institute of Electrical \& Electronics Engineers (IEEE), Orlando, Florida, USA, 2012). doi:10.1109/infcom.2012.6195456

14. P Li, S Guo, S Yu, AV Vasilakos, Reliable multicast with pipelined network coding using opportunistic feeding and routing. IEEE Trans. Parallel Distributed Syst. 25(12), 3264-3273 (2014). doi:10.1109/tpds.2013.2297105

15. Intelligent Transport System (ITS); Vehicular Communications; Basic Set of Applications; Definition, ETSI specification TR 102 638, v.1.1.1 (2009). http://www.etsi.org/. Accessed 22 April 2016

16. R Chen, W-L Jin, A Regan, Broadcasting safety information in vehicular networks: issues and approaches. IEEE Netw. 24(1), 20-25 (2010). doi:10.1109/mnet.2010.5395779

17. A Rahim, FB Muhaya, M Sher, ZS Khan, I Ahmad, Performance evaluation of broadcast techniques in VANETs. Indian J. Sci. Technol. 2, 52-55 (2009). doi:10.17485/ijst/2009/v2i10/30721

18. K Obraczka, KViswanath, G Tsudik, Flooding for reliable multicast in multi-hop ad hoc networks. Wireless Netw. 7(6), 627-634 (2001). doi:10.1023/a:1012323519059

19. Y Mylonas, M Lestas, A Pitsillides, P loannou, V Papadopoulou, Speed adaptive probabilistic flooding for vehicular ad hoc networks. IEEE Trans. Vehicular Technol. 64(5), 1973-1990 (2015). doi:10.1109/tvt.2014.2339316

20. N Wisitpongphan, OK Tonguz, JS Parikh, P Mudalige, F Bai, V Sadekar, Broadcast storm mitigation techniques in vehicular ad hoc networks. IEEE Wireless Commun. 14(6), 84-94 (2007). doi:10.1109/mwc.2007.4407231

21. AY Al-Dubai, MB Khalaf, W Gharibi, J Ouenniche, in 2015 IEEE 81st Vehicular Technology Conference (VTC Spring). A new adaptive probabilistic broadcast protocol for vehicular networks (Institute of Electrical \& Electronics Engineers (IEEE), Glasgow, Scotland, UK, 2015), pp. 1-5. doi:10.1109/vtcspring.2015.7145627

22. A Mohammed, M Ould-Khaoua, LM Mackenzie, J-D Abdulai, in 2nd International Conference on Adaptive Science \& Technology (ICAST). Dynamic probabilistic counter-based broadcasting in mobile ad hoc networks (Institute of Electrical \& Electronics Engineers (IEEE), Accra, Ghana, 2009), pp. 120-127. doi:10.1109/icastech.2009.5409735

23. M Bakhouya, J Gaber, P Lorenz, An adaptive approach for information dissemination in vehicular ad hoc networks. J. Netw. Comput. Appl. 34(6), 1971-1978 (2011). doi:10.1016/j.jnca.2011.06.010

24. MB Yassein, SF Nimer, AY Al-Dubai, A new dynamic counter-based broadcasting scheme for mobile ad hoc networks. Simul. Modell. Pract. Theory. 19(1), 553-563 (2011). doi:10.1016/j.simpat.2010.08.011

25. D Li, H Huang, X Li, M Li, F Tang, in International Conference on Wireless Communications, Networking and Mobile Computing (WiCom). A distance-based directional broadcast protocol for urban vehicular ad hoc network (Institute of Electrical \& Electronics Engineers (IEEE), Shanghai, China, 2007), pp. 1520-1523. doi:10.1109/wicom.2007.383

26. G Korkmaz, E Ekici, F Ozguner, Black-burst-based multihop broadcast protocols for vehicular networks. IEEE Trans. Vehic. Technol. 56(5), 3159-3167 (2007). doi:10.1109/tvt.2007.900493

27. FJ Ros, PM Ruiz, I Stojmenovic, in IEEE 69th Vehicular Technology Conference (VTC-Spring). Reliable and efficient broadcasting in vehicular ad hoc networks (Institute of Electrical \& Electronics Engineers (IEEE), Barcelona, Spain, 2009), pp. 1-5. doi:10.1109/vetecs.2009.5073834

28. KN Nakorn, K Rojviboonchai, Non-GPS data dissemination for VANET. Int. J. Distributed Sensor Netw. 2014, 1-17 (2014). doi:10.1155/2014/906084

29. M Li, K Zeng, W Lou, Opportunistic broadcast of event-driven warning messages in vehicular ad hoc networks with lossy links. Comput. Netw. 55(10), 2443-2464 (2011). doi:10.1016/..comnet.2011.04.005

30. S Gonzalez, V Ramos, in 9th International Conference on Next Generation Mobile Applications, Services and Technologies (NGMAST). Fast-OB-VAN: a fast opportunistic broadcast protocol for VANETs (Institute of Electrical \& Electronics Engineers (IEEE), Cambridge, United Kingdom, 2015), pp. 114-119. doi:10.1109/ngmast.2015.52

31. B Blaszczyszyn, A Laouiti, P Muhlethaler, Y Toor, in 8th International Conference on ITS Telecommunications. Opportunistic broadcast in VANETs (OB-VAN) using active signaling for relays selection (Institute of Electrical \& Electronics Engineers (IEEE), Phuket, Thailand, 2008), pp. 384-389. doi:10.1109/itst.2008.4740291
32. D Kim, C-K Toh, J-C Cano, P Manzoni, in IEEE Wireless Communications and Networking, (WCNC). A bounding algorithm for the broadcast storm problem in mobile ad hoc networks (Institute of Electrical \& Electronics Engineers (IEEE), New Orleans, LA, USA, pp. 1131-1136. doi:10.1109/wcnc.2003.1200530

33. O Tonguz, N Wisitpongphan, F Bai, DV-CAST: a distributed vehicular broadcast protocol for vehicular ad hoc networks. IEEE Wireless Commun. 17(2), 47-57 (2010). doi:10.1109/mwc.2010.5450660

34. Microscopic and Continuous Road Traffic Simulator, Simulation of Urban MObility (SUMO). http://sumo-sim.org. Accessed 22 April 2016

35. Network Simulator 3 (NS-3): Discrete Event Network Simulator. http:// nsnam.org. Accessed 22 April 2016

36. J Bu, G Tan, N Ding, M Liu, C Son, in Proceedings of the Workshop on ns-3 (WNS3), May 2014, Atlanta GA, USA. Implementation and evaluation of WAVE 1609.4/802.11p in ns-3. doi:10.1145/2630777.2630778

\section{Submit your manuscript to a SpringerOpen ${ }^{\circ}$ journal and benefit from:}

- Convenient online submission

Rigorous peer review

- Immediate publication on acceptance

- Open access: articles freely available online

- High visibility within the field

- Retaining the copyright to your article

Submit your next manuscript at $\boldsymbol{\nabla}$ springeropen.com 